\title{
Retrospective clinical analysis of adverse drug reactions associated with antiretroviral therapy in Tlokwe district, South Africa
}

\author{
Rentia van Graan ${ }^{\mathrm{a}}$, Michelle Viljoen ${ }^{\mathrm{a} *}$, Malie Rheeders $^{\mathrm{a}}$ and Fadeela Motara ${ }^{\mathrm{b}}$ \\ ${ }^{a}$ Centre of Excellence for Pharmaceutical Sciences (PharmaCen), Division of Pharmacology, School of Pharmacy, North-West University, \\ Potchefstroom, South Africa \\ 'Potchefstroom Hospital, Pharmacy, Potchefstroom, South Africa \\ ${ }^{*}$ Corresponding author, email: mviljoen@uwc.ac.za

Background: South Africa has the highest number of patients on antiretroviral therapy (ART) globally. Various obstacles were identified that influence effective reporting of adverse drug reactions (ADRs) in resource-limited countries. This investigation aimed to identify, classify and analyse the prevalence of ART-related ADRs.

Methods:This observational, quantitative and retrospective descriptive investigation utilised ADR forms completed by healthcare professionals in various healthcare facilities in the Tlokwe district, South Africa (January 2010 to December 2014). Descriptive and inferential analyses were carried out.

Results: A total of 770 ART-related ADRs were included in the final analysis. The mean age was 40.1 ( $\pm 10.1 \%)$ years, with significantly higher ADRs reported in females (70.8\%). In this study, 99\% of the ADRs were reported by doctors. Abnormal fat distribution (58\%), peripheral neuropathy $(21.6 \%)$ and renal dysfunction $(6.6 \%)$ were most frequently reported. Females presented with abnormal fat distribution and peripheral neuropathy at a significantly younger age ( $38.1 \pm 4.6$ vs. $43.4 \pm 5.7$ years, $p<0.0001$ and $39.7 \pm 1.1$ vs. $45.1 \pm 9.2$ years, $p<0.001$ ) respectively compared with males. Gender difference was practically significant (Cramer's $V=0.3$ ) for all three of the major reported ADRs.

Conclusions: Gender was highly dependent among the major reported ADR categories, and women presented with abnormal fat distribution and peripheral neuropathy at a significantly earlier age than males. This retrospective analysis can serve as a platform for future ADR studies within this district. Sustainable and continuous efforts should be made to train and create more awareness among healthcare workers in this district.

Keywords: antiretroviral therapy, adverse drug reactions, drug safety, pharmacovigilance, gender differences

\begin{abstract}
Introduction
The WHO defined pharmacovigilance as the science and activities that relate to 'detection, assessment, understanding and prevention of adverse effects or any other possible drug related problems.' It can be described as a dynamic umbrella term, which holds the key to effective ADR monitoring in practice to minimise ADRs and benefit patient populations. ${ }^{2}$
\end{abstract}

In South Africa, the Medicines Control Council (MCC) and the National Pharmacovigilance Centre (NPC), as part of the National Department of Health (NDoH), facilitate the reporting of ADRs by health professionals, the management of safety data which arise during clinical trials, spontaneous reporting and post-marketing surveillance. ${ }^{3}$ Public health pharmacovigilance promotes risk minimising of preventable ADRs, whereas institutional or clinical pharmacovigilance targets hospitals and clinics aiming to reduce mortality and morbidity associated with ADRs. ${ }^{4}$

Surveys conducted on the status of pharmacovigilance in South Africa confirmed that the communication and analysis of safety data could be improved by collective strengthening efforts in all current and future programmes. ${ }^{4}$ The problematic occurrence of widespread underreporting of ADRs and the importance of addressing shortcomings effectively in pharmacovigilance activities in the public healthcare setting need to be appropriately addressed. ${ }^{5}$

Furthermore, knowledge regarding antiretroviral (ARV) toxicity in developing countries is limited. These toxicities can result in unknown long-term effects and compromise patient confidence and adherence. ${ }^{6,7}$
With the highest prevalence of human immunodeficiency virus (HIV) infection worldwide, South Africa's ART programme consumes the biggest part of the South African pharmacovigilance resources. ${ }^{5}$ The morbidity and mortality caused by this epidemic are well known globally. ${ }^{8}$ The recent Global AIDS update indicated that 3.4 million people in South Africa were on ART, making it the country with the highest number of patients on ART compared with the 17 million globally at the end of $2015 .{ }^{9}$

Patients with epidemic diseases, such as tuberculosis (TB) and HIV, are at an increased risk of experiencing ADRs that can be attributed to the safety profile of concomitant complex ARV and anti-TB regimens and the compromised immune system of these patients. The rollout of the ART programme in South Africa highlighted the significance of targeted monitoring of the safety of medications used in patients and the development of targeted pharmacovigilance systems to address and solve the particular problems of medications commonly used in ARV regimens. ${ }^{6}$

Currently in the South African public sector, the majority of ADRs are reported spontaneously. These data are collectively employed through the national pharmacovigilance database to enable change in national health policies if and when required. ${ }^{10}$

Collaboration between the Department of Pharmacology (NorthWest University) and Potchefstroom Hospital Pharmacy was initiated to conduct an internal quality improvement project to retrospectively investigate, analyse and classify all the reported ADRs from the Tlokwe district. The focus, however, for this paper is on the prevalence of antiretroviral-related ADRs only. 


\section{Methods \\ Setting and design}

In this study, the ADR forms were studied retrospectively from those already completed by healthcare professionals in various healthcare facilities within the Tlokwe district, from January 1 , 2010 to December 31, 2014. The Tlokwe district is one of four local municipalities within the Dr Kenneth Kaunda District in the North West Province. Potchefstroom Hospital is the main site for the distribution of medicines to the respective Tlokwe areas, which includes nine primary healthcare clinics. The inclusion criteria for this analysis were reports from January 1, 2010 to December 31, 2014, documentation regarding the date of birth, date on which treatment was discontinued if the ADR so required and signature of a registered healthcare professional. Report forms were excluded from this analysis if they were used to indicate regimen changes due to virological failure, pregnancy, incorrect reporting due to out-phasing of stavudine, downreferral to clinics or patient medication non-adherence.

Ethics approval was granted by the Human Research Ethics Committee of the NWU Potchefstroom Campus (NWU-0003115-A1) and by the Department of Health, North West Province, during November 2015. This study was conducted and adhered to according to the principles of the Declaration of Helsinki.

\section{Data collection}

The following information was captured from completed ADR forms into an electronic database: age, gender, hospital or clinic setting, date and reporter (doctor, pharmacist or registered nurse) of the ADR, date of onset of the ADR, name of suspected drug(s) responsible for the ADR and the type of ADR reported. Data were recorded using only one electronic data-collection tool in the form of a set-designed Excel ${ }^{\circ}$ (Microsoft Corp, Redmond, WA, USA) spreadsheet. Patient anonymity was maintained by removing any personal identifiers prior to capturing into the electronic database. The forms were numbered from 1 onwards, as they were captured, and these numbers were removed once data integrity and validity were completed. To establish integrity and validity of the electronically captured data, an independent person randomly selected $20 \%$ of the captured ADR forms to double check their accuracy.

\section{Statistical analysis}

Descriptive statistics, mean (standard deviation $( \pm S D)$ ) and median (minimum and maximum) were used to describe and summarise the clinical and demographic characteristic (Table 1). The Mann-Whitney test was used to compare age differences (see Figure 3) and Pearson's chi-square test was applied to investigate dependence of categorical data, such as gender, between the three most frequently reported ADRs. Statistical significance was regarded as $p<0.05$. Statistica ${ }^{\circledR}$ version 13.0 (StatSoft, Tulsa, OK, USA) was used and consultative guidance was provided by a statistician from North-West University, Potchefstroom Campus.

\section{Results}

A total of 1,295 ADR forms, from January 1, 2010 to December 31, 2014, were evaluated and 1,195 reported ADR cases (some forms indicated more than one ADR per form) were further subjected to the set inclusion and exclusion criteria. This resulted in the exclusion of $5.2 \%(n=62)$ due to incomplete data, regimen changes due to virological failure $(14.4 \%, n=172)$, pregnancy $(2.6 \%, n=31)$ or down-referral to clinics, patient non-compliance or stavudine being phased out $(12.7 \%, n=152)$.
Table 1: Patient demographics recorded for reported ADRs during 2010-2014

\begin{tabular}{|c|c|}
\hline Characteristic & Result \\
\hline Total number of ADRs & 778 \\
\hline Number of ADRs related to ARVs, $n$ (\%) & $770(99.0)$ \\
\hline Male, $n(\%)$ & $191(24.6)$ \\
\hline Female, $n(\%)$ & $551(70.8)$ \\
\hline Gender not specified, $n(\%)$ & $36(4.6)$ \\
\hline Age (years), mean (SD) & $40.1 \pm 10.1$ \\
\hline Age (years), median (min; max) & $40(5.0 ; 73.0)$ \\
\hline \multicolumn{2}{|l|}{ Age categories, $n(\%)$} \\
\hline Children: $\leq 18$ years & $16(2.1)$ \\
\hline Young adults $18.1-30$ years & $109(14.0)$ \\
\hline Middle aged adults: $31-59$ years & $634(81.5)$ \\
\hline Elderly: $\geq 60$ years & $18(2.3)$ \\
\hline Age not specified, $n(\%)$ & $1(0.1)$ \\
\hline \multicolumn{2}{|l|}{ Number of reported ADRs per facility, $n(\%)$} \\
\hline Hospital & $363(46.7)$ \\
\hline Clinic & $121(15.6)$ \\
\hline Facility not specified & $294(37.8)$ \\
\hline \multicolumn{2}{|l|}{ Number of reported ADRs per HCP, $n(\%)$} \\
\hline Doctor & $769(98.8)$ \\
\hline Registered professional nurse & $8(1.0)$ \\
\hline HCP not specified & $1(0.1)$ \\
\hline
\end{tabular}

Notes: ADRs: adverse drug reactions; ARVs: antiretrovirals; HCP: healthcare professional.

Figure 1 illustrates the number of collected ADR forms and how they were assessed to derive the total number of ADRs $(n=778)$ included in this analysis. Antiretroviral-related ADRs made up the largest portion $(99 \%, n=770)$ with only $1 \%(n=8)$ of the reported ADRs not related to ARVs during this five-year period concurrently.

The majority of ADRs $(71.5 \%, n=556)$ were reported during 2011 and the least number $(0.6 \%, n=5)$ during 2010 . There was a decrease over time in the reported ADRs (2012: $n=112,14.4 \%$; 2013: $n=64,8.2 \%$; and 2014: $n=41,5.3 \%)$. Surprisingly, only $0.7 \%$ of patients were TB-HIV co-infected and received ART and TB treatment concurrently.

Table 2: Reported adverse drug reactions according to gender

\begin{tabular}{lccc}
\hline Type of ADRs & Female & Male & $\begin{array}{c}\text { Gender not } \\
\text { specified }\end{array}$ \\
\hline Abnormal fat distribution, $n(\%)$ & $358(46)$ & $77(9.9)$ & $16(2.1)$ \\
\hline Peripheral neuropathy, $n(\%)$ & $97(12.5)$ & $61(7.8)$ & $10(1.3)$ \\
\hline Renal dysfunction, $n(\%)$ & $23(3.0)$ & $25(3.2)$ & $3(0.4)$ \\
\hline Blood abnormalities, $n(\%)$ & $12(1.5)$ & $10(1.3)$ & $2(0.3)$ \\
\hline Gynecomastia, $n(\%)$ & $2(0.3)^{*}$ & $8(1.0)$ & $2(0.3)$ \\
\hline Hyperlactatemia, $n(\%)$ & 0 & $8(1.0)$ & 0 \\
\hline CNS abnormalities, $n(\%)$ & $12(1.5)$ & $5(0.6)$ & $2(0.3)$ \\
\hline Skin abnormalities, $n(\%)$ & $12(1.5)$ & $2(0.3)$ & $1(0.13)$ \\
\hline Gl abnormalities, $n(\%)$ & $6(0.8)$ & $1(0.1)$ & 0 \\
\hline Lactic acidosis, $n(\%)$ & $15(1.9)$ & $1(0.1)$ & 0 \\
\hline Diverse ADRs, $n(\%)$ & $6(0.8)$ & $1(0.1)$ & 0 \\
\hline
\end{tabular}

Notes: ADRs: adverse drug reactions; *breast hypertrophy; CNS: central nervous system; Gl: gastrointestinal. 


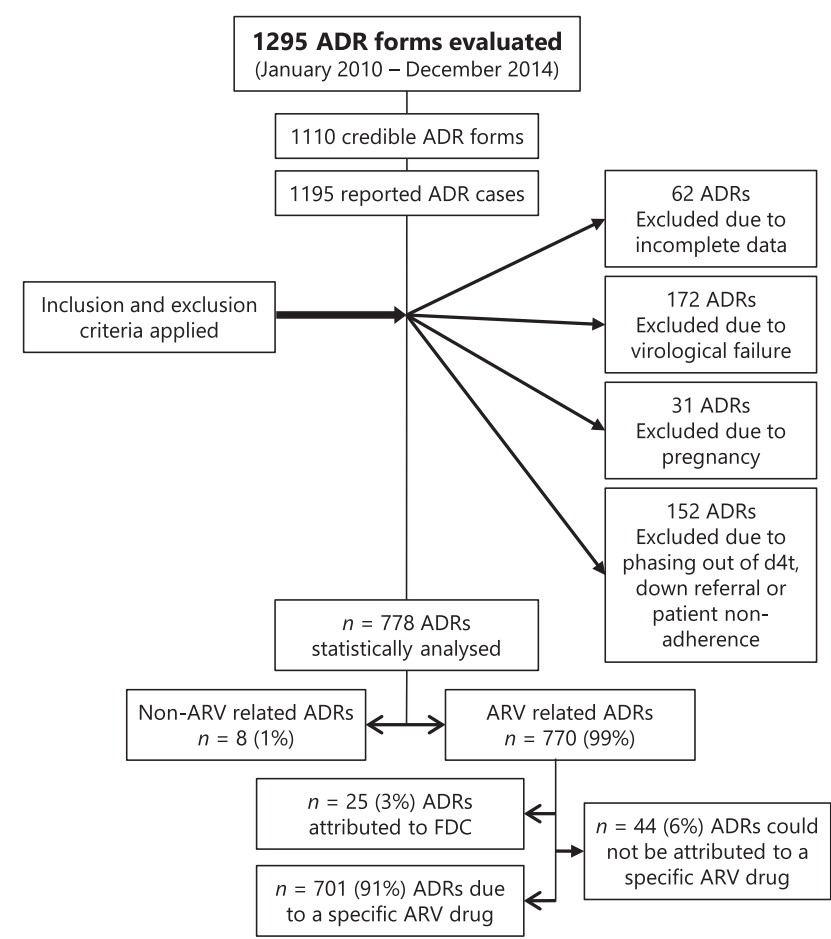

Figure 1: Flow diagram of data collected from already reported ADR forms.

Notes: ADR: adverse drug reaction, d4t: stavudine, ARV: antiretroviral, FDC: fixeddose combination.

Table 1 presents the patient demographic data, indicating $70.8 \%$ of ADRs were reported in females with the mean total population age being $40.1( \pm 10.1 \%)$ years.

\section{ADRs reported by gender}

The ADRs were classified into 11 categories, of which the frequencies by category and according to gender are reflected in Table 2. The three most reported ADRs were abnormal fat distribution (58\%, $n=451$ ), peripheral neuropathy $(21.6 \%$, $n=168)$ and renal dysfunction $(6.6 \%, n=51)$. Abnormal fat distribution comprised the highest frequency of ADRs in women (46\%, $n=358)$ compared with men $(9.9 \%, n=77)$, followed by peripheral neuropathy in women $(12.5 \%, n=97)$ and in men
$(7.8 \%, n=61)$ and third, renal dysfunction with a slight tendency to occur more in men $(3.2 \%, n=25)$ compared with women $(3.0 \%, n=23)$. The highest frequencies in eight of the 11 defined categories were reported in females.

\section{ADRs reported by healthcare professionals}

Significantly, more doctors (98.8\%) reported ADRs compared with only $0.9 \%$ of the professional nurses (Table 3 ). During this period, no pharmacists reported any ADRs. The majority of ADRs reported were in the hospital setting $(46.7 \%, n=363)$ compared with clinics $(15.6 \%, n=121)$.

\section{ADRs and associated $A R V s$}

The frequencies of the respective ARVs responsible for the reported ADRs are summarised in Figure 2. Stavudine $(\mathrm{d} 4 \mathrm{t})$ was clearly the ARV associated with the most $(74.2 \%, n=577)$ reported ADRs. This was followed by a combination of three or more ARV drugs $(5.7 \%, n=44)$, TDF most commonly known to cause renal toxicity $(5.0 \%, n=39)$, AZT the major cause of anaemia and peripheral neuropathy in susceptible patients $(4.4 \%, n=34)$, FDCs $(3.2 \%, n=25)$, NVP responsible for inducing liver abnormalities $(3.1 \%, n=24)$ and EFV responsible for central nervous system associated side effects $(2.2 \%, n=17)$. Adverse drug reactions in which no drug causality was reported comprised $5.7 \%(n=44)$ (not reflected in Figure 2).

\section{ADRs and stratification according to age and gender}

The gender comparison of the three major reported ADR categories related to age are depicted in Figure 3. There was a significant $(p<0.0001)$ difference in the age of females compared with males ( $38.1 \pm 4.95$ vs. $43.4 \pm 5.66$ years), for whom abnormal fat distribution was reported. The same trend was observed for peripheral neuropathy $(45.10 \pm 9.19$ vs. $39.74 \pm 1.41, p-0.0011)$; however, no significant $(p=0.66)$ difference was found between the ages in the reporting of renal dysfunction.

Additional analysis with Pearson's chi-square test indicated statistically significant $(p<0.0001)$ dependence between genders for all three reported categories (renal dysfunction, abnormal fat distribution and peripheral neuropathy), as well as medium practical significance with a Cramer's $V$-value of 0.28 .

Table 3: Distribution of ADRs reported by healthcare professionals in different facilities

\begin{tabular}{|c|c|c|c|c|c|c|}
\hline \multirow[t]{2}{*}{ Type of ADR } & \multicolumn{3}{|c|}{ Healthcare professional } & \multicolumn{3}{|c|}{ Facility } \\
\hline & RPN & Doctor & Not specified & Hospital & Clinics & Not specified \\
\hline Abnormal fat distribution, $n$ (\%) & $5(0.6)$ & $446(57.3)$ & 0 & $215(27.6)$ & $55(7.1)$ & $181(23.3)$ \\
\hline Peripheral neuropathy, $n$ (\%) & 0 & $168(21.6)$ & 0 & $65(8.4)$ & $26(3.3)$ & $77(9.9)$ \\
\hline Renal dysfunction, $n$ (\%) & $1(0.1)$ & $50(6.4)$ & 0 & $29(3.7)$ & $14(1.8)$ & $8(1.0)$ \\
\hline Blood abnormalities, $n$ (\%) & 0 & $24(3.1)$ & 0 & $13(1.7)$ & $6(0.8)$ & $5(0.6)$ \\
\hline CNS abnormalities, $n(\%)$ & 0 & $19(2.4)$ & 0 & $12(1.5)$ & $4(0.5)$ & $3(0.4)$ \\
\hline Lactic acidosis, $n$ (\%) & 0 & $16(2.1)$ & 0 & $9(1.2)$ & $4(0.5)$ & $3(0.4)$ \\
\hline Skin abnormalities, $n(\%)$ & $1(0.1)$ & $13(1.7)$ & $1(0.1)$ & $8(1.0)$ & $4(0.5)$ & $3(0.4)$ \\
\hline Gynecomastia, $n$ (\%) & 0 & $12(1.5)$ & 0 & $4(0.5)$ & $5(0.6)$ & $3(0.4)$ \\
\hline Hyperlactatemia, $n$ (\%) & 0 & $8(1.0)$ & 0 & 0 & 0 & $8(1.0)$ \\
\hline Gl abnormalities, $n$ (\%) & 0 & $7(0.9)$ & 0 & $5(0.6)$ & $1(0.1)$ & $1(0.1)$ \\
\hline Diverse, $n(\%)$ & $1(0.1)$ & $6(0.8)$ & 0 & $3(0.4)$ & $2(0.3)$ & $2(0.3)$ \\
\hline Total & $8(0.9)$ & $769(98.8)$ & $1(0.1)$ & $363(46.7)$ & $121(15.6)$ & $294(37.8)$ \\
\hline
\end{tabular}

Notes: RPN: registered professional nurse; CNS: central nervous system; GI: gastrointestinal. 


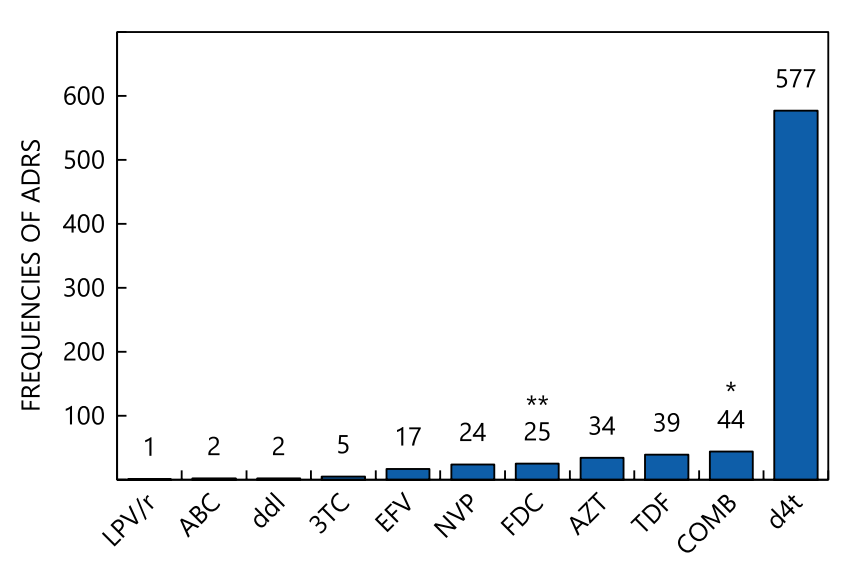

CAUSATIVE DRUG

Figure 2: Frequencies of adverse drug reactions associated with drug causality.

Notes: LPV/r: lopinavir/ritonavir combination; ABC: abacavir; ddl: didanosine, 3TC: lamivudine; EFV: efavirenz; NVP: nevirapine; FDC: fixed-dose combination; AZT: zidovudine; TDF: tenofovir disoproxil fumarate; d4t: stavudine. * ${ }^{*}$ COMB: Any combination of three or more ARV drugs; ** FDC: TDF+ 3TC+EFV.

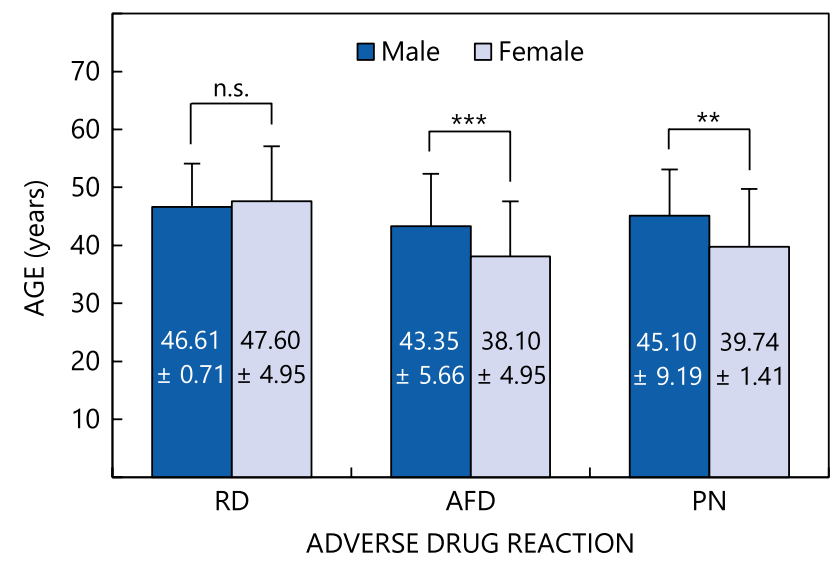

Figure 3: Gender comparison of the mean age $( \pm S D)$ in terms of the highest reported ADRs.

Notes: RD: renal dysfunction; AFD: abnormal fat distribution; PN: peripheral neuropathy. n.s: not significant, ${ }^{* *} p<0.001,{ }^{* * *} p<0.0001$.

\section{Discussion}

This study aimed to describe the frequencies of reported ADRs related to ARVs and, more specifically, to characterise the clinical impact on patients as South Africa has the largest number of people on ARVs globally. ${ }^{9}$

The mean age (SD) of these patients was $40.1( \pm 10.1)$ years. There was a higher rate of ADR occurrences (81.5\%) in middle aged adults (31-59 years) compared with individuals 30 years old or younger, which is in agreement with other recent studies in South Africa. ${ }^{11,12}$ Gender differences played a profound role as more than two-thirds (70.8\%) of ADRs were reported in women compared with men $(24.6 \%)$ in this study. This gender variance in reporting ARV-related ADRs was also observed in other African settings, where ADR reporting was investigated in public healthcare settings with regard to gender differences, incidence, type and risk factors for ADRs. ${ }^{13-15}$ The observation that ADRs are more prevalent in women was supported in data collected from 590 patients ${ }^{11}$ from different provinces (Gauteng, Limpopo and Mpumalanga) in South Africa by the Medunsa National ARV Pharmacovigilance Surveillance System, during January 2007 to
August 2011 and also in another study conducted in Mpumalanga during July 2011 to February $2013 .^{8}$ Women account for a large proportion (estimated $47 \%$ ) of the world's HIV population with about 18.6 million currently infected ${ }^{16}$ and, furthermore, they are an emergent concern due to findings in several observational studies that point towards greater and recurrent toxic effects of ARVs in the female population. ${ }^{17}$

Pharmacovigilance activities within this district prior to and during 2010 were inattentive, insufficient and possibly responsible for under-reporting of ADRs. Focused training sessions and establishment of local pharmacovigilance cluster groups were set in place and organised by Potchefstroom Hospital during 2011. This may explain the very small number (0.6\%) of ADRs reported during 2010 compared with over twothirds (71.5\%) in 2011 , following the awareness and training that took place. However, the numbers of reported ADRs continuously waned from 2012 to 2015. Improved ADR reporting led to improvement in drug assessments and resulted in earlier discovery of severe adverse reactions in a study conducted amongst 300 nurses in an Iranian hospital, after an educational intervention was made to improve their knowledge and attitude regarding ADR reporting. ${ }^{18}$ An extensive systemic review to assess the effectiveness of different strategies to improve ADR reporting concluded that multiple interventions, including constant training of healthcare professionals, ultimately improved ADR reporting rates. ${ }^{19}$

In this retrospective study, medical doctors reported $98.8 \%$ of the ADRs compared with only $0.9 \%$ by professional registered nurses, and the majority (46.6\%) of ADRs were reported within the hospital setting compared with $15.6 \%$ from the clinic settings. It has been shown in other studies that educational interventions can improve physician awareness and reporting of ADRs, and these physicians were able to use the knowledge gained from close-up training in daily practices. ${ }^{20}$ Gupta and Udupa highlighted the benefit of from long-term reporting of ADRs when correct ADR reporting is coupled with closer interaction between healthcare professionals and pharmacovigilance centres. ${ }^{21}$

The most prevalent ADRs reported in this retrospective study were abnormal fat distribution (58\%), peripheral neuropathy (21.6\%) and renal dysfunction (6.6\%). The high frequency of abnormal fat distribution was similar to the early stavudine era, ${ }^{22-24}$ but decreased over time in correlation with the phasing out of stavudine, post-2010. Peripheral neuropathy (19\%), lipodystrophy (18.9\%) and renal failure (5.6\%) associated with ARVs were reported in a study conducted over a similar period and were more representative of the geography of South Africa. ${ }^{12}$ It is interesting to note that a much lower incidence for abnormal fat distribution (6\%) during 2007 to 2012 was reported by MEDUNSA National ARV Pharmacovigilance Centre when stavudine was still frequently used as part of first-line regimens (1a \& 1b)..$^{11}$

Similar peripheral neuropathy frequencies (25\%, 21\% and 20\%) to this study were reported in three other studies in Malawi, Cameroon and South Africa, after patients were initiated on stavudine-containing regimens. ${ }^{8,11,15,25}$

Renal dysfunction related ADRs were fewer (2.3\%) in HIV-infected patients in Lesotho compared with this study (6.6\%). ${ }^{26}$ Higher CNS-related ADRs (9.9\%), ${ }^{15}$ and even more significantly higher incidences of CNS symptoms (27.4\%) and anaemia (16.1\%), were 
reported in a self-reported cross-sectional study $(n=384)$ at a university hospital in Gondar, Ethiopia, on adult patients receiving $\mathrm{ART}^{27}$ compared with our study (2.4\%).

Prior to 2010, the ADR forms used in South Africa were product complaint forms with no separate allocated space for the reporting of ARV-related ADRs. After 2010, the forms were specifically designed to indicate ADRs caused by ARVs, which may explain the much higher percentage of reported ADRs due to ARVs $(98.9 \%)$, but not the low frequency $(0.7 \%)$ of reported clinical manifestations in TB-HIV co-infected patients in this study.

The most frequent causative drug in this study was stavudine (74.2\%), which was responsible for the majority of abnormal fat distribution manifestations, particularly during 2011. Stavudine was previously identified and implicated as the cause of abnormal fat distribution, peripheral neuropathy, hyperlactatemia and lactic acidosis in various investigations in South Africa since 2007. ${ }^{11,12,28}$ Globally, stavudine was phased out but only slowly in South Africa from 2010 and more vigorously since 2013, correlating with the drastic decrease in reported ADRs noted between 2011 and 2012, which was also evident from our study.

HIV-infected patients on ART are at greater risk for age-associated toxicities. ${ }^{29}$ In this study, females presented, on average, 5 years younger with abnormal fat distribution and peripheral neuropathy compared with males. It is further evident that $75 \%$ of the patients in this study, who experienced the three major ADRs, were $\geq 31$ years, which is also supported by other studies. ${ }^{11,30,31}$

In this study, gender was not only statistically significant but also practically significant $(V=0.28)$ in patients who reported the three most frequent ADRs (abnormal fat distribution, peripheral neuropathy and renal dysfunction). Among these HIV-infected adults, women were more likely to present with abnormal fat distribution compared with men. These results are in agreement with reports by Ofotokun and Pomeroy ${ }^{13}$ and Ofotokun. ${ }^{17}$ The gender differences with regard to the frequency of renal dysfunction were not significant; furthermore, men who did report renal-related ADRs seemed to experience it at a slightly younger mean age (46.6 vs. 47.6 years) compared with women. However, other studies have shown that women are predisposed to experiencing ADRs more frequently when receiving NRTIs such as AZT and stavudine. . $^{13,15,32}$

Limitations of this study included its retrospective and spontaneous nature, which formed part of this internal quality improvement project. The data incorporated were restricted to previously completed ADR forms over the stated 5 years' duration. No further investigations into laboratory results or other diagnostic tests were pursued and no differentiation between the different grades of ADRs or medication adherence was made during this retrospective analysis. The data were only representative of ADR reports from public healthcare facilities in the Tlokwe district. These ADR forms were also used to indicate regimen changes due to pregnancy, virological failure or when down-referred to clinics. Collective and decisive steps should be taken on a process for how to handle these other scenarios where regimen changes or down-referrals are made which are not due to ADRs.

\section{Conclusion}

The most frequently reported ADRs were abnormal fat distribution, peripheral neuropathy and renal dysfunction in both women and men. Women experienced significantly more abnormal fat distribution and peripheral neuropathy that occurred at a significantly younger age compared with men. The importance of this study is that it contributed to the knowledge of this previously under-researched geographic area, and identified patients predisposed to risk factors for ADRs. Modifiable risk factors may thus be addressed early. The results on stavudine, predominantly responsible for the abnormal fat distribution, retrospectively supports its phasing out as published in the South African guidelines in 2010 to 2013.

This retrospective investigation may form a reference platform with which Tlokwe district reported ADRs post-December 2014 should be compared, especially if future research or similar quality improvement projects are to be undertaken. It is imperative that continuous and sustainable efforts be made to create awareness, to improve knowledge through training and to report ADRs correctly within this district.

Disclosure statement - The authors declare that they have no conflicts of interest.

\section{References}

1. World Health Organization (WHO): The importance of pharmacovigilance. Safety monitoring of medicinal products. 2002 [cited 2017 February 9]. https://apps.who.int/medicinedocs/pdf/ s4893e/s4893e.pdf.

2. Jeetu G, Anusha G. Pharmacovigilance: a worldwide master key for drug safety monitoring. J Young Pharm. 2010;2: 315-2. https://doi.org/10.4103/0975-1483.66802

3. Medicines Control Council: Reporting of post-marketing adverse drug reactions to human medicinal products in South Africa. 2016. [cited 2017 March 7]. https://www.mccza.com/documents/f39361792.33 ADR_reporting_post-marketing_Jul16_v4.1_showing_changes.pdf

4. Mehta U, Dheda M, Steel G, Blockman $M$, et al. Strengthening pharmacovigilance in South Africa. SAMJ. 2014;104: 104-6.

5. Ruud KW, Srinivas SC, Toverud E-L. Addressing gaps in pharmacovigilance practices in the antiretroviral therapy program in the Eastern Cape Province. South Africa. Res Social Adm Pharm. 2010;6: 345-53. https://doi.org/10.1016/j.sapharm.2009.11.006

6. World Health Organization (WHO): Pharmacovigilance for antiretrovirals in resource-poor countries. 2007 [cited 2017 February 7]. https://www.who.int/hiv/pub/pharmacovigilance/resource-poor/ en/.

7. Kenny J, Musiime V, Judd A, Gibb D. Recent advances in pharmacovigilance of antiretroviral therapy in HIV-infected and exposed children. Curr Opin HIV AIDS. 2012;7: 305-16. https://doi.org/10.1097/COH.0b013e328354da1d

8. Dheda M, Distefano K, Sunduzwayo K, et al. Decentralized HIV/AIDS pharmacovigilance in South Africa: Mpumalanga success \& moving forward. J AIDS HIV Res. 2013;5: 370-9.

9. Joint United Nations Programme on HIV/AIDS (UNAIDS): Global AIDS update. 2016 [cited 2017 March 4]. https://www.unaids.org/sites/ default/files/media_asset/global-AIDS-update-2016_en.pdf.

10. Dheda M. Decentralized HIV/AIDS pharmacovigilance in South Africa: Mpumalanga as pilot province for national roll-out. J AIDS HIV Res. 2013;5: 357-65.

11. Masenyetse LJ, Manda SO, Mwambi HG. An assessment of adverse drug reactions among HIV positive patients receiving antiretroviral treatment in South Africa. AIDS Res Ther. 2015;12(6): 1-8. doi:10.1186/ s12981-015-0044-0.

12. Birbal $S$, Dheda $M$, Ojewole $E$, et al. Adverse drug reactions associated with antiretroviral therapy in South Africa. Afr J AIDS Res. 2016;15(3): 243-8. 
13. Ofotokun I, Pomeroy C. Sex differences in adverse reactions to antiretroviral drugs. Top HIV Med. 2003;11: 55-9.

14. Eluwa Gl, Badru T, Akpoigbe KJ. Adverse drug reactions to antiretroviral therapy (ARVs): incidence, type and risk factors in Nigeria. BMC Pharmacol Toxicol. 2012;12(1): 7. doi:10.1186/14726904-12-7.

15. Luma HN, Doualla M-S, Choukem S-P, et al. Adverse drug reactions of highly active antiretroviral therapy (HAART) in HIV infected patients at the General Hospital, Douala, Cameroon: a cross sectional study. Pan Afr Med J. 2012;12: 87. doi:10.11604/pamj.2012.12.87.1438.

16. The United Nations Children's Fund: The AIDS epidemic continues to take a staggering toll, especially in sub-Saharan Africa. 2016 [cited 2017 March 10]. Available from https://data.unicef.org/topic/hivaids/ global-regional-trends/.

17. Ofotokun I. Sex differences in the pharmacologic effects of antiretroviral drugs: potential roles of drug transporters and phase 1 and 2 metabolizing enzymes. Top HIV Med. 2005;13: 79-83.

18. Hanafi S, Torkamandi H, Hayatshahi A, et al. An educational intervention to improve nurses' knowledge, attitude, and practice toward reporting of adverse drug reactions. Iran J Nurs Midwifery Res. 2014;19: 101-6.

19. Gonzalez-Gonzalez C, Lopez-Gonzalez E, Herdeiro MT, et al. Strategies to Improve Adverse Drug Reaction Reporting: A Critical and Systematic Review. Drug Safety. 2013;36: 317-28. https://doi.org/10.1007/s40264-013-0058-2

20. Tabali M, Jeschke $E$, Bockelbrink $A$, et al. Educational intervention to improve physician reporting of adverse drug reactions (ADRs) in a primary care setting in complementary and alternative medicine. BMC Public Health. 2009;9(1): 1200. doi:10.1186/1471-2458-9-274.

21. Gupta P, Udupa A. Adverse drug reaction reporting and pharmacovigilance: Knowledge, attitudes and perceptions amongst resident doctors. J Pharm Sci Res. 2011;3: 1064-1069.

22. Carr A, Cooper DA. Adverse effects of antiretroviral therapy. Lancet. 2000;356: 1423-30. https://doi.org/10.1016/S0140-6736(00)02854-3

23. Ys DM, Smita DM. Adverse drug reactions (ADR) due to anti-retrovirals (ARV): issues and challenges. Indian J Sex Transm Dis. 2005;26: 40-9.

24. Leclercq P, Goujard C, Duracinsky $M$, et al. High prevalence and impact on the quality of life of facial lipoatrophy and other abnormalities in fat tissue distribution in hiv-infected patients treated with antiretroviral therapy. AIDS Res Hum Retrov. 2013;29: 761-8. https://doi.org/10.1089/aid.2012.0214
25. Kampira E, Kumwenda J, van Oosterhout JJ, Dandara C. Mitochondria DNA subhaplogroups L0a2 and L2a modify susceptibility to peripheral neuropathy in Malawian adults on stavudine containing highly active antiretroviral therapy. J Acquir Immune Defic Syndr. 2013;63: 647-52. https://doi.org/10.1097/QAI.0b013e3182968ea5

26. Maja LJ. Assessment of adverse drug reactions caused by HAART at antiretroviral clinics in the Maseru district, Lesotho [DissertationMaster degree]. North-West University; 2014.

27. Tadesse WT, Mekonnen AB, Tesfaye WH, et al. Self-reported adverse drug reactions and their influence on highly active antiretroviral therapy in HIV infected patients: a cross sectional study. BMC Pharmacol Toxicol. 2014;15. doi:10.1186/2050-6511-15-32.

28. Maskew M, Westreich D, Fox MP, et al. Effectiveness and safety of $30 \mathrm{mg}$ versus $40 \mathrm{mg}$ stavudine regimens: a cohort study among HIV-infected adults initiating HAART in South Africa. J Int AIDS Soc. 2012;15(1): 13. doi:10.1186/1758-2652-15-13.

29. Effros RB, Fletcher CV, Gebo K, et al. Workshop on HIV infection and aging: what is known and future research directions. Clin Infect Dis. 2008;47: 542-53. https://doi.org/10.1086/591953

30. Mugomeri E, Olivier D, van den Heever WM. The effect of tenofovir on renal function in HIV-positive patients in Lesotho. Med Tech S. 2014;28: 34-8.

31. Yu YM, Shin WG, Lee JY, et al. Patterns of adverse drug reactions in different age groups: analysis of spontaneous reports by community pharmacists. PLOS ONE. 2015;10(7): e0132916. doi:10.1371/journal. pone.0132916.

32. Singh H, Dulhani N, Tiwari $P$, et al. A prospective, observational cohort study to elicit adverse effects of antiretroviral agents in a remote resource-restricted tribal population of Chhattisgarh. Indian J Pharmacol. 2009;41: 224-6. https://doi.org/10.4103/0253-7613.58512

Received: 25-04-2017 Accepted: 31-07-2017 